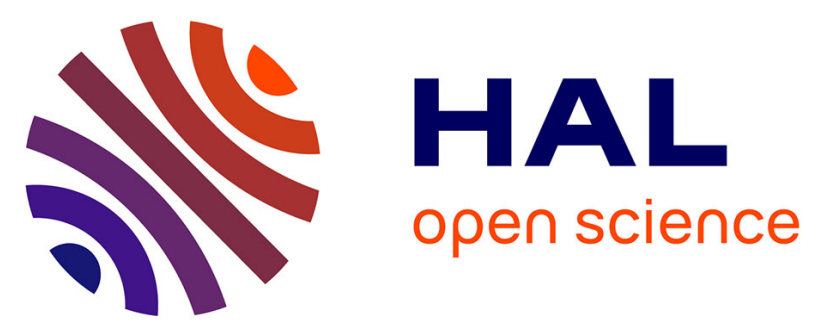

\title{
How coatings with hydrophobic particles may change the drying of water droplets: incompressible surface versus porous media effects
}

Benoit Laborie, Florent Lachaussée, Elise Lorenceau, Florence Rouyer

\section{- To cite this version:}

Benoit Laborie, Florent Lachaussée, Elise Lorenceau, Florence Rouyer. How coatings with hydrophobic particles may change the drying of water droplets: incompressible surface versus porous media effects. Soft Matter, 2013, pp.1. 10.1039/C3SM50164G . hal-00807741

HAL Id: hal-00807741

https://hal-enpc.archives-ouvertes.fr/hal-00807741

Submitted on 4 Apr 2013

HAL is a multi-disciplinary open access archive for the deposit and dissemination of scientific research documents, whether they are published or not. The documents may come from teaching and research institutions in France or abroad, or from public or private research centers.
L'archive ouverte pluridisciplinaire HAL, est destinée au dépôt et à la diffusion de documents scientifiques de niveau recherche, publiés ou non, émanant des établissements d'enseignement et de recherche français ou étrangers, des laboratoires publics ou privés. 


\title{
Soft Matter
}

RSCPublishing

\section{How coatings with hydrophobic particles may change the drying of water droplets: incompressible surface versus porous media effects}

\author{
Benoît Laborie, $^{\text {ac }}$ Florent Lachaussée, $^{a}$ Elise Lorenceau ${ }^{a}$ and Florence Rouyer ${ }^{\star b c}$ \\ There is no clear statement on the role of particles in the drying of liquid marbles, which are liquid drops \\ coated with hydrophobic solid particles. While some works report a similar drying time for liquid marbles \\ and bare water drops others observe a faster evaporation of either liquid marbles or of bare water drops. \\ To provide insight into the subject, we report water drying experiments in different configurations. We first \\ focus on the drying of flat water surfaces coated with a single or several layers of hydrophobic micronic \\ particles. Quite surprisingly, surfaces coated with a single layer of densely packed particles dry at the \\ same speed as the bare surfaces. However, when coated with several layers of particles, the drying rate \\ per unit surface area is significantly diminished. This effect is quantitatively explained by considering \\ vapor diffusion through the porous media formed by the stacking of micronic particles above the \\ interface. Then, we consider the drying of curved interfaces which are liquid marbles, i.e. drops coated \\ with one monolayer of micronic particles. Those systematically dry faster than pure drops of the same \\ initial volume. As the presence of a single layer of particles does not significantly affect the drying rate, \\ this "speed-up" effect is attributed to the conservation of the surface area of the coated drop during \\ the drying. Our quantitative experiments and understanding of the drying of liquid marbles therefore \\ support the different results found in the literature: liquid marbles coated with one monolayer of fine \\ solid particles do dry faster than water drops, while those coated with several layers - that may be \\ formed by aggregates of nanoparticles - experience slower drying.
}

Received 15th January 2013

Accepted 27th February 2013

DOI: $10.1039 / \mathrm{c3sm50164g}$

www.rsc.org/softmatter depends on the wetting properties (pinned or unpinned contact line and angle of contact). For droplets with a pinned contact line, $\mathrm{Hu}$ and Larson ${ }^{9}$ showed that the drying rate is constant for a low initial contact angle (less than $45^{\circ}$ ) whereas it decreases with time for a large initial contact angle. This time dependence of the drying is confirmed by recent experiments at ambient temperature. ${ }^{10,11}$ For a droplet with a non-pinned contact line, the drying rate is characterized by the " $D$ law". ${ }^{12}$ As the liquid dries at the air-liquid interface, the lifetime of a droplet $\tau$ is proportional to its interface area, which scales as $D^{2}$, where $D$ is the droplet diameter. Thus, the " $D^{2}$ law" can be written as: $D^{2}(t)=8 j_{0}\left(t_{\mathrm{f}}-t\right)$, where $j_{0}$ has the dimension of a diffusion coefficient and depends on temperature and humidity of the atmosphere. In the usual ambient atmosphere $j_{0}$ is expected to be equal to $2.5 \times 10^{-10} \mathrm{~m}^{2} \mathrm{~s}^{-1}$, thus the lifetime of a $10 \mu \mathrm{m}$ diameter droplet is typically $50 \mathrm{~ms} .^{12}$

Liquid marbles, the surfaces of which are covered to $90 \%$ with solid particles, are thus promising candidates for microfluidics applications, as the particles may hinder the drying. In particular, if the rate of water loss were proportional to the uncovered surface fraction, then the lifetime of a liquid marble should be 10 times longer than one of the bare liquid droplets. Unfortunately, this naive idea is wrong: ${ }^{13,14}$ the lifetimes of bare
${ }^{a}$ Université Paris-Est, Laboratoire Navier (UMR CNRS 8205, École des Ponts ParisTech, IFSTTAR), 2, allée Kepler, 77420 Champs-sur-Marne, France

${ }^{b}$ Université Paris-Est, Laboratoire Navier (UMR CNRS 8205, École des Ponts ParisTech, IFSTTAR), 5 bd Descartes, 77454 Marne-la-Vallée, France

${ }^{c}$ Université Paris-Est, Laboratoire L.P.M.D.I (EA 7264), 5 bd Descartes, 77454 Marnela-Vallée, France.E-mail: florence.rouyer@univ-mlv.fr 
droplets and liquid marbles are of the same order of magnitude. More precisely, the experimental results from the literature do not reach a clear consensus on the subject: the mass loss during drying of liquid marbles seems to depend on the experimental set-up, size and nature of the particles. First, at high temperature (i.e. above the Leidenfrost temperature), Aberle et al. reported that the drying times of bare water droplets and liquid marbles coated with graphite particles are almost the same, thus suggesting a similar drying flux for the two systems. ${ }^{1}$ Yet, using the same graphite particles, Dandan and Erbil, who deduced the mass loss of liquid marbles during drying from image analysis and assuming a spherical shape for the drop, demonstrated a slower drying of liquid marbles. ${ }^{3}$ An identical result was obtained for liquid marbles coated with microsized polytetrafluoroethylene particles (PTFE). ${ }^{4}$ With these PTFE particles, the authors mentioned a possible aggregation of particles during the drying: the liquid marbles may be coated with an increasing number of particle layers that may explain the dramatic slowdown of the drying rate. Finally, measuring the drying rate with a high precision thermogravimetric analyzer, Bhosale et al. reached similar conclusions: liquid marbles coated with fumed silica nanoparticles may experience a longer or the same lifetime as bare water drops. ${ }^{2}$ Here also, such liquid marbles coated with nanoparticles are almost entirely transparent. It is therefore impossible to determine - in a simple way - whether they are coated by a single or several layers of particles. However, in the same work, the authors also reported that liquid marbles coated with microsized PTFE particles experience a shorter lifetime than a bare water drop. This speed-up of the drying is surprising, as these microparticles are similar to those used by Tosun and Erbil, ${ }^{4}$ for which the drying rate was diminished. The speed-up is attributed by Bhosale et al. to the wrinkled shape of the coated drops observed at the end of the drying: while the volume of liquid of the droplet is forced to decrease, the surface concentration of the particles - that are irreversibly absorbed to the interface increases. This induces a high surface pressure that hinders the area reduction due to water loss. For high coverage of particles, the interface cannot sustain this stress and buckles inward. ${ }^{15-18}$ The deformations of liquid marbles bound by this quasi solidlike shell are thus comparable to the invagination and buckling of a drying droplet made of a colloidal suspension. ${ }^{19,20}$ Despite this important framework and while the buckling of liquid marbles has been clearly established, there is no quantitative understanding of its influence on the drying rate. Moreover, one question remains unanswered: why do liquid marbles coated with micro or nanosized particles have such different lifetimes? In particular, does it depend on the number of layers of the particles or the size of the particles?

The present work strives to give a quantitative understanding of the drying of liquid marbles. We first experimentally show that the number of particle layers above the interface can significantly decrease the drying rate. Moreover, by considering vapor diffusion through porous media, we quantitatively relate the drying rate reduction to the height and porosity of the packing independently of the particle size. This porous media effect can explain the slowdown of the drying rate previously reported for nanoparticles, ${ }^{2}$ which easily form thick aggregates. Then, we quantitatively analyze the importance of buckling. We show that the drying rate of coated drops with a monolayer of microparticles is constant - its mass decaying linearly with time - which is in agreement with the model of drying by diffusion based on a constant surface area of the drop.

\section{Experimental: materials and measurements}

The particles used in this study are made of polystyrene (PS) purchased from Microbeads AS with a density $(\rho)$ equal to $1.05 \mathrm{~g}$ $\mathrm{cm}^{-3}$. We use three different sets of monodisperse beads with sharp Gaussian distributions. The three mean diameters $(d)$ are: $40 \mu \mathrm{m}, 140 \mu \mathrm{m}$ and $500 \mu \mathrm{m}$ and the standard deviations are typically less than $10 \%$ for the three distributions. To make them more hydrophobic, the particles were chemically modified by silanization. For $20 \mathrm{~g}$ of particles, we typically used $100 \mathrm{ml}$ of octane containing $0.1 \mathrm{~g}$ of FDTCS (perfluorodecyltrichlorosilane ABCR GmbH \& Co. KG). Hydrophobic fumed silica nanoparticles (R709) purchased from Degussa were also used, the particle diameter and density are respectively equal to $40 \mathrm{~nm}$ and $2 \mathrm{~g} \mathrm{~cm}^{-3}$.

\section{Flat coated interface}

To obtain flat interfaces covered with particles, we gently deposit PS particles at the interface of a cylindrical beaker filled with water (Millipore). Two vessels with different diameters $\Delta$ are used $(\Delta=51.6 \mathrm{~mm}$ or $33.4 \mathrm{~mm})$. Depending on the mass of particles ( $\left.m_{\text {grains }}\right)$, we either obtain mono or multilayers of particles. The surface fraction of the monolayer measured by visualization was equal to $93 \pm 1 \%$ which is slightly larger than the value of hexagonal close packing (90.6\%). This may be due to particles that overlap with one another as a premise of formation of the second layer of particles. The number of layers $(n)$ is determined by measuring the thickness of the grains packing which is related to the mass of grains by: $H=4 m_{\text {grains }} /$ $\left(\rho \pi \Delta^{2}(1-\phi)\right)$ where $\phi$ is the porosity of the porous media (see Fig. 1a) and $n=H / d$. From visualization of $H$, we deduce that $\phi=0.4$, which is in agreement with measurements of loose packing density of frictional grains in air. ${ }^{21,22}$

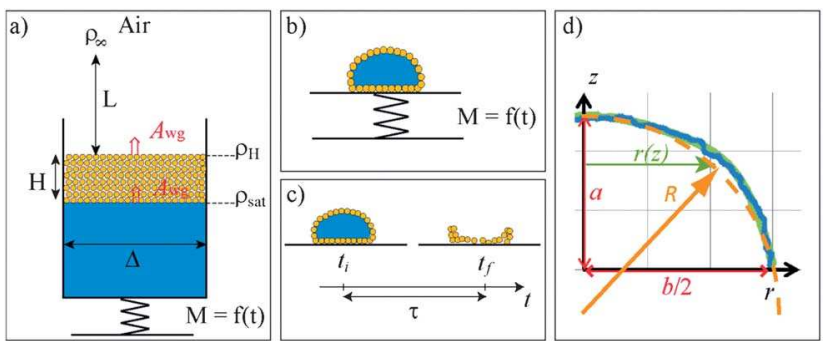

Fig. 1 Different experimental set-ups. (a) Mass measurement of a vessel with a flat water interface coated with a single or several layers of particles. (b) Mass measurement of a liquid marble as a function of time. (c) Measurement of the drying time of a liquid marble. (d) Profile of a drop that illustrates its height $a$ and its diameter $b$ of the surface of contact with the horizontal plane. 


\section{Curved coated interface: liquid marbles}

The liquid marbles are obtained by rolling water drops on a stack of PS particles up to a state where no more particles can be trapped at the surface of the drop. The initial pure water (MilliQ) drops are millimetric. They are deposited on a horizontal hydrophobic plate that was obtained by sticking hydrophobic rough colored particles of hundred micrometers on to a glass plate as shown in Fig. 2 . The contact angle between the drops and the substrate is of the order of $90^{\circ}$. The coverage of the surface is high typically $92 \pm 2 \%$ (inset of Fig. 2) as for the monolayer at a flat interface. The evolution of the liquid marble during drying was followed using a digital camera and a mirror providing simultaneously top and side views of the drop (see Fig. 2). The frequency acquisition of the camera $f$ is typically $0.017 \mathrm{~s}^{-1}$. The characteristics of the liquid marble before drying (initial volume and surface) are deduced from the digital images assuming symmetry of the marble around vertical axis. Two methods are used to calculate the water volume of the drop. The first one consists of measuring the height $(a)$ of the drop and the diameter $(b)$ of the surface in contact with the horizontal plane (cf. Fig. 1d) and then approximating the shape of the drop using a hemisphere with the same height and the same base surface. For each drop, we calculated the radius of curvature $=\frac{b^{2}}{8 a}+\frac{a}{2}$, the surface $S=2 \pi R a$ and the volume $V=\pi\left(R^{2} a-\frac{1}{3}\left(R^{3}-(R-a)^{3}\right)\right)$. The second method consists of extracting the profile $r(z)$ (see Fig. 1d) and integrating over the surface: $S=\int_{0}^{a} 2 \pi r(z) \mathrm{d} z$ and the volume $V=\int_{0}^{a} \pi(r(z))^{2} \mathrm{~d} z$. The deviation from one method to the other is less than $10 \%$ on average. For both methods, the volume of water $\left(V_{\mathrm{w}}\right)$ inside the
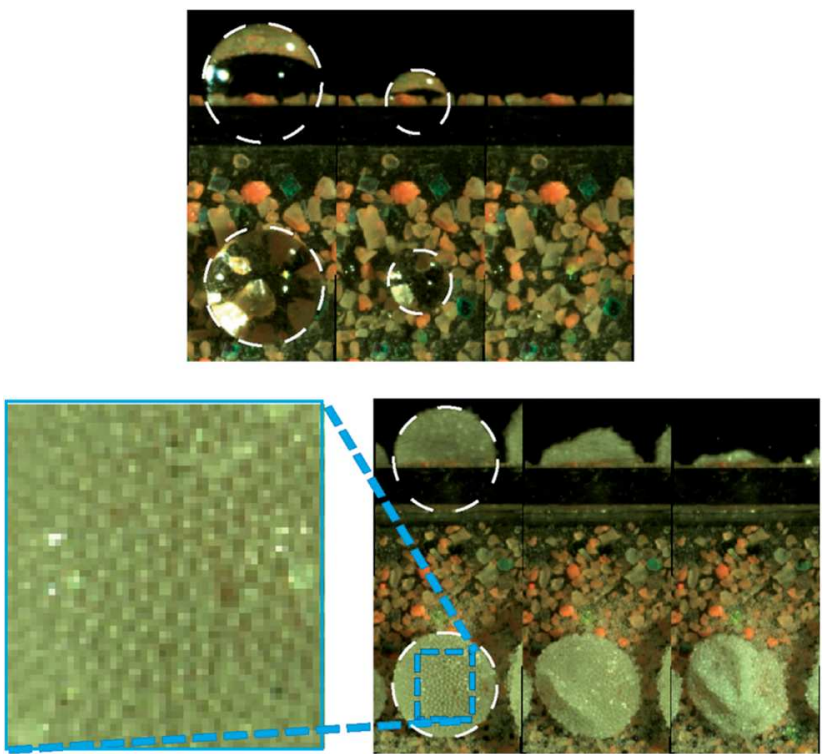

Fig. 2 Top and side views of the water droplets at initial, middle-life and final times for a bare interface and for a interface armored with particles of $140 \mu \mathrm{m}$ diameter. The initial volume of the droplet is equal to $14.6 \pm 1.4 \mathrm{~mm}^{3}$. The dotted white circles are a guide for the eye to highlight the spherical shape of the drops. drop is approximated by $V_{\mathrm{w}}=V-S\left(\frac{d}{2}\right)$ where $V$ is the volume of the drop and $S\left(\frac{d}{2}\right)$ is half of the volume of the particle monolayer.

The drying experiments were performed in an ambient atmosphere. For each experiment, we measured the temperature $T$ and relative humidity $\mathrm{RH}\left(T=21^{\circ} \mathrm{C} \pm 1{ }^{\circ} \mathrm{C}\right.$ and $\mathrm{RH}=$ $50 \% \pm 3 \%$ ). To follow the kinetics of drying of coated interface, we use two distinct experimental set-ups.

First, the mass of a vessel or of a liquid marble $(M)$ is recorded using a balance as a function of time (see Fig. 1a and b). The slope gives the drying rate $A$ in $\mathrm{g} \mathrm{s}^{-1}$. The precision on mass measurement is $10^{-4} \mathrm{~g}$ and the time interval between each acquisition can be varied from $1 \mathrm{~s}$ up to one hundred seconds. During one experiment, the mass of the sample decreases by at least of $5 \times 10^{-3} \mathrm{~g}$ and thus the incertitude of $A$ is at maximum equal to $2 \%$. To gain insight into the role of particles in drying, we follow the mass loss of two samples: one with a bare water interface and one with an interface covered with particles. The initial masses of the two samples are slightly different: thus by placing alternately one sample after the other on the balance, we simultaneously measure the mass loss of the two samples under the same experimental conditions.

We also measure the time $(\tau)$ of drying that we named "drying time" for liquid marbles of different initial volumes. The drying time is the difference between the initial time $\left(t_{\mathrm{i}}\right)$ and final time of drying $\left(t_{\mathrm{f}}\right)$ which are determined as follows. The initial time of drying is the onset of buckling of liquid marbles (once the liquid marble starts drying, its shape deviates from a hemisphere) while the final time corresponds to the instant when no water is observed. In that case, there is no more variation of intensity between two consecutive images. This yields a typical precision on $\tau$ of $\pm 2 / f= \pm 2$ minutes, where $f$ is the acquisition frequency of the camera.

\section{Results and modeling}

\section{Drying of flat coated interface}

Typical mass variations $(M)$ with time are reported in Fig. 3 for a vessel with diameter $\Delta=51.6 \mathrm{~mm}$ coated with particles of

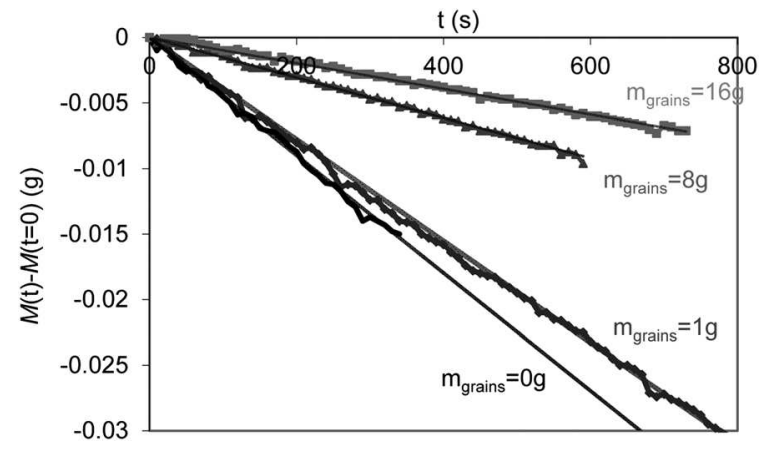

Fig. 3 Mass variation of water in a vessel $(\Delta=51.6 \mathrm{~mm})$ as a function of time for different masses of particles. The mass decreases linearly with time yielding a constant drying rate $A_{w g}$. 
$80 \mu \mathrm{m}$ diameter. We compare drying through four different packings $\left(m_{\text {grains }}=0 \mathrm{~g} ; 1 \mathrm{~g} ; 8 \mathrm{~g}\right.$ and $16 \mathrm{~g}$ ) which correspond to four heights of the grain ranging from 0 to $12 \mathrm{~mm}$. The masses of the filled vessels linearly decrease with time, thus yielding a constant drying rate $A$. Moreover $A$ decreases as $m_{\text {grains }}$ or $H$ increases. Fig. 4a displays the drying rate of vessels with an interface covered with multilayers of particles $\left(A_{\mathrm{wg}}\right)$ normalized by the drying rate of bare water $\left(A_{\mathrm{w}}\right)$ as a function of $n$, the number of particle layers for two different particle diameters $(d=140 \mu \mathrm{m}$ and $d=80 \mu \mathrm{m})$, in a vessel with diameter $\Delta=$ $51.6 \mathrm{~mm}$. Quite logically, $A_{\mathrm{wg}} / A_{\mathrm{w}}$ decays with the number of layers $n$ : the drying is hindered by the presence of thick porous media above the interface. We also note that for the same number of layers, the normalized drying rate of the coated interface decreases when the particle diameter increases. We stress that the drying rates of the bare water interface and of an interface covered with a single layer of particles are almost the same: The presence of a single layer of particles does not alter significantly the drying rate.

To quantify how thick porous media can hinder drying, we first recall the classical features of the drying by diffusion at a bare flat water interface. The rate of water loss $A_{\mathrm{w}}$ is analogous to the drying rate of a sessile drop in contact with a solid
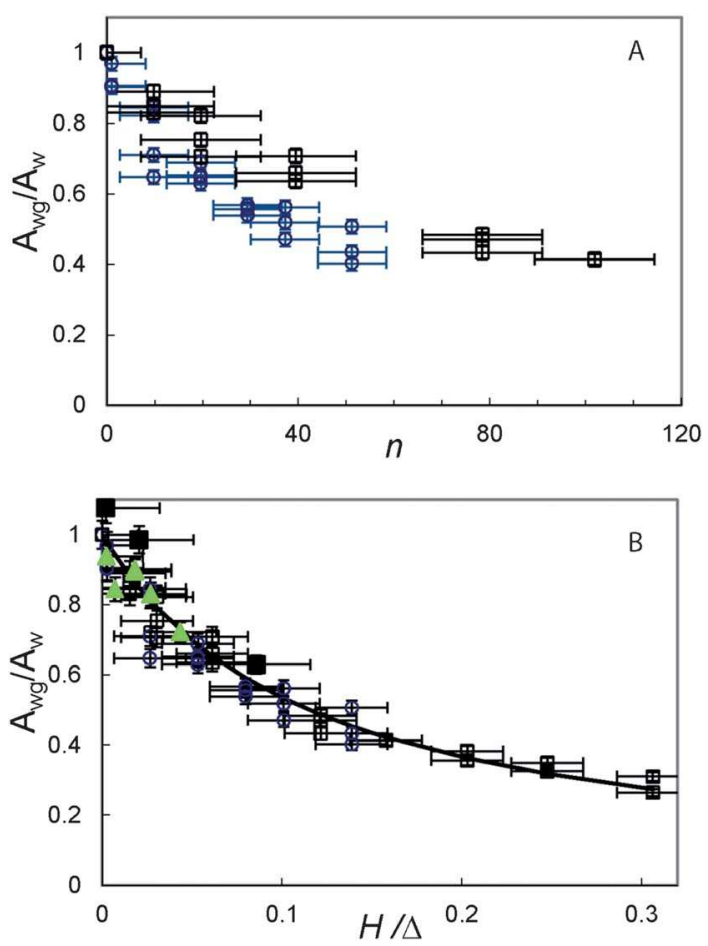

Fig. 4 (A) Normalized water loss rate of an interface covered with multilayers of hydrophobic particles $\left(A_{w g} / A_{w}\right)$ as a function of the number of layers in a cylindrical vessel of diameter $\Delta=51.6 \mathrm{~mm}$. Circles correspond to PS particles with $d=$ $140 \mu \mathrm{m}$ while squares correspond to $d=80 \mu \mathrm{m}$. (B) Normalized water loss rate of an interface covered with multilayers of hydrophobic particles with $d=140 \mu \mathrm{m}$ (open circles), $80 \mu \mathrm{m}$ (squares) and $40 \mathrm{~nm}$ (triangles) as a function of the thickness of the porous media normalized by its diameter. Open and full symbols correspond respectively to vessels with diameter $\Delta=51.6 \mathrm{~mm}$ and $\Delta=33.4 \mathrm{~mm}$. The continuous line corresponds to the analytical prediction given by eqn (1), based on vapor diffusion in dry porous media as explained in the text. surface $\pi \Delta^{2}$ and with angle of contact $\theta$ that tends to zero. ${ }^{12}$ In this framework, $A_{\mathrm{w}}$ is equal to: $A_{\mathrm{w}}=2 D_{\mathrm{m}} \Delta\left(\rho_{\mathrm{sat}}-\rho_{\infty}\right)$ where $D_{\mathrm{m}}$ is the molecular diffusion coefficient, $\rho_{\text {sat }}$ is the saturation concentration of water vapor in air and $\rho_{\infty}$ is the concentration of water vapour in the laboratory. $A_{\mathrm{w}}$ can also be written as a classical diffusion equation introducing the gradient of concentration of water vapor in air over a typical distance $L$ (see Fig. 1) where $L=\pi \Delta / 8$ such as: $A_{\mathrm{w}}=\frac{\pi \Delta^{2}}{4} D_{\mathrm{m}} \frac{\left(\rho_{\text {sat }}-\rho_{\infty}\right)}{L}$.

To take into account the particle layers, we use a theoretical approach which has been developed to understand the drying of a granular packing. ${ }^{23}$ The vapor diffusion rate through the porous media $\left(A_{\mathrm{wg}}\right)$ of thickness $H$ toward the open air depends on the concentration of water vapor above the porous media $\rho_{\mathrm{H}}$ and the saturation concentration just above the interface as sketched in Fig. 1, yielding: $A_{\mathrm{wg}}=\frac{\pi \Delta^{2}}{4} \psi D_{\mathrm{m}} \frac{\left(\rho_{\mathrm{sat}}-\rho_{\mathrm{H}}\right)}{H}$. In this equation, $\psi$ is the correction factor for the diffusion coefficient relative to the porosity $\phi$ and the tortuosity of the porous media. It is written as $\psi=\phi^{4 / 3}$ for an unsaturated packing of spheres. ${ }^{24}$ Then, considering vapor diffusion in air yields: $A_{\mathrm{wg}}=\frac{\pi \Delta^{2}}{4} D_{\mathrm{m}} \frac{\left(\rho_{\mathrm{H}}-\rho_{\infty}\right)}{L}$. Eventually, vapor flow continuity implies: $A_{\mathrm{wg}}=A_{\mathrm{w}} \frac{1}{1+8 H / \psi \pi \Delta}$.

Experimentally, we measure that $\phi$, the porosity of the packing of the hydrophobic grain, is equal to 0.4. This yields $\psi=\phi^{4 / 3}=0.29$, thus giving:

$$
\frac{A_{\mathrm{wg}}}{A_{\mathrm{w}}}=\frac{1}{1+8.64 H / \Delta}
$$

In Fig. $4 \mathrm{~B}, A_{\mathrm{wg}} / A_{\mathrm{w}}$ is reported as a function of $H / \Delta$ for four different particle diameters and two vessel diameters. We observe an excellent collapse of the data for the different particle and vessel diameters. Moreover, the data are perfectly adjusted by eqn (1) with no adjustable parameter (continuous line). A linear regression analysis on experimental data gives $\frac{1}{\left(\frac{A_{\mathrm{wg}}}{A_{\mathrm{w}}}\right)}-1=(8.4 \pm 0.4) H / \Delta$. This regression is in very good agreement with the value of eqn (1) based on vapor diffusion through porous media assuming that the expression of the correction factor $\psi=\phi^{4 / 3}$.

Drying rate decays with $H / \Delta$ and is halved for $H / \Delta$ of order 0.1. This model shows that the rate of water loss is independent of the particle size and only depends on the ratio of the thickness of porous media over the typical diameter of the air-water interface.

Moreover, eqn (1) predicts that the water loss rate through a porous media with a contact diameter $\Delta=51 \mathrm{~mm}$ and thickness $H$ around $100 \mu \mathrm{m}$, which corresponds to a single layer of particles, is 0.98 (very close to 1 ) in agreement with the data. 

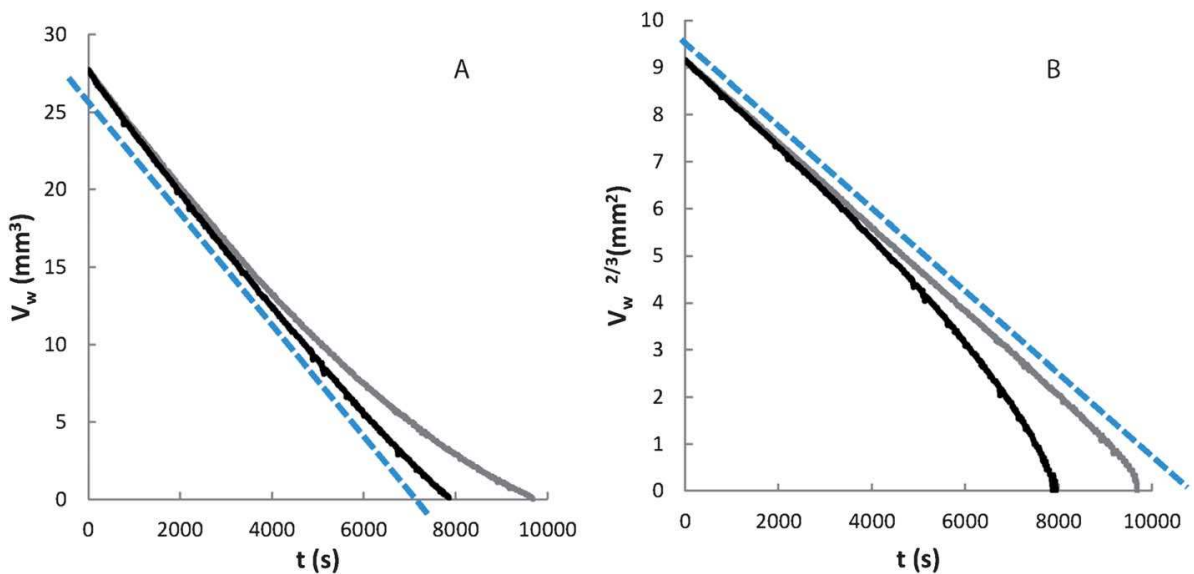

Fig. 5 (A) Water volume $\left(V_{w}\right)$ of the drop as a function of time deduced from weight measurements assuming that the density of water is equal to $10^{3} \mathrm{~kg}^{-3}$. (B) Same data but $V_{\mathrm{w}}{ }^{2 / 3}$ as a function of time. Bare interface (grey) and interface covered with particles of $140 \mu \mathrm{m}$ diameter (black). The blue dotted line is a guide for the eye.

\section{Drying of curved coated interface}

Bearing in mind that a single layer of particles on a flat water interface does not significantly alter the water loss rate, we now come to the drying of liquid marbles. We first report (Fig. 5A) the volume variations with time of a bare and a coated drop in an ambient atmosphere. Under the same experimental conditions, a bare water drop of $25 \mathrm{~mm}^{3}$ dries in $9700 \mathrm{~s}$, while the same liquid volume coated with a single layer of PS particles with $140 \mu \mathrm{m}$ diameter dries in $7900 \mathrm{~s}$. Moreover, volume variations of the bare drop suggests a slowdown of drying with time, whereas the volume of coated drops decays linearly with time. In Fig. 5B, data for a bare droplet show a linear decay of $V_{\mathrm{w}}{ }^{2 / 3}$ with time which qualitatively agrees with the $D^{2}$ law mentioned in the introduction for purely diffusive evaporation of droplet with a non-pinned contact line. Indeed, as shown in Fig. 1, the contact line is not pinned for a bare droplet. In contrast, for coated droplets, the contact line is pinned with an initial contact angle roughly equal to $90^{\circ}$, the drying rate is constant whereas $\mathrm{Hu}$ and Larson predicted a decrease of drying rate with time for an initial contact angle larger than $45^{\circ}$. Quantitative modeling of these data is provided in Fig. 5 .

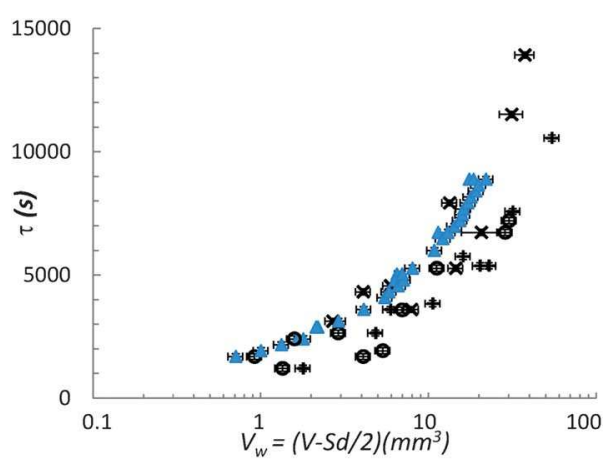

Fig. 6 Drying time $\tau$ as a function of $V_{w}$ for different interfaces, measured by visualization: bare interface (blue triangles); interfaces armored by particles with $d=40 \mu \mathrm{m}(O), 140 \mu \mathrm{m}(\times)$ and $500 \mu \mathrm{m}(+)$.
We plot in Fig. $6 \tau$ the drying time of coated and bare drops as a function of $V_{\mathrm{w}}$, the initial water volumes of the drops. The drying time of liquid marbles is scattered. Yet, for a given volume, it seems to be slightly smaller than for bare water drops in agreement with our mass measurements.

These results are in contradiction with those of paragraph I, which demonstrate that the particles barely hinder the drying. Thus, a liquid marble should dry at the same rate (or a slightly slower rate if there are several layers of particles) as a bare droplet. To understand this significant slowdown of water loss, we successively consider drying by diffusion of bare sessile drops and liquid marbles.

For a bare water droplet, the local evaporative diffusive flux for sessile drops of radius $R$ is radial and is written as: ${ }^{12} j=j_{0} / R$ where $j_{0}=D_{\mathrm{m}} \frac{\left(\rho_{\mathrm{sat}}-\rho_{\mathrm{H}}\right)}{\rho_{\mathrm{L}}}$ is the evaporation parameter that depends on the molecular diffusion coefficient $D_{\mathrm{m}}$ and on the relative humidity of the atmosphere. Thus, the total evaporative flux is written as: $\frac{\mathrm{d} V}{\mathrm{~d} t}=-\frac{j_{0}}{R} S(t)$ where volume $\left(V(t)=\alpha R(t)^{3}\right)$ and surface area $\left(S(t)=\beta R(t)^{2}\right)$ of a spherical cap are respectively proportional to $R^{3}$ and $R^{2}$ with $\alpha$ and $\beta$ for geometrical constants. It becomes $3 \alpha R(t) \frac{\mathrm{d} R}{\mathrm{~d} t}=-j_{0} \beta$. After integration, this leads to

$$
V(t)^{2 / 3}=\alpha^{2 / 3} R(t)^{2}=V_{\mathrm{i}}^{2 / 3}-\left(\frac{2 j_{0}}{3} \beta \alpha^{-1 / 3}\right) t
$$

and

$$
\tau=\frac{3}{2 j_{0}} \frac{V_{\mathrm{i}} R_{\mathrm{i}}}{S_{\mathrm{i}}}
$$

where the subscript i corresponds to values at initial time.

Eqn (2) can be quantitatively compared to the experimental value of water mass loss during drying. By visualizating various bare water drop profiles, we deduced the values of $\alpha$ and $\beta$ (Fig. 7 left). Then, we analyze the mass measurement data and plot $\left(V^{2 / 3}-V_{\mathrm{i}}^{2 / 3}\right)$ versus time (Fig. 7 right). This quantity 


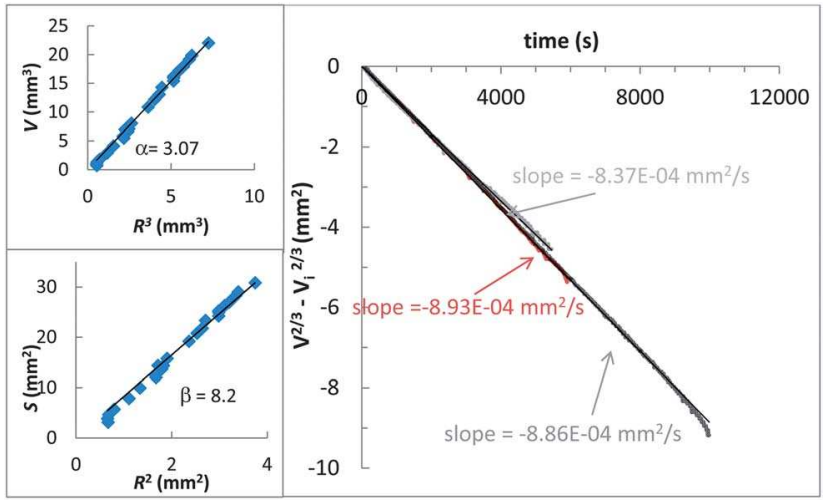

Fig. 7 Left: geometrical factor of a bare droplet: volume (top) and surface (bottom) as a function of radius of curvature; right: variation of in volume calculated from mass measurement of a drop during drying for three bare drops of different initial volumes: $\left(V^{2 / 3}-V_{i}^{2 / 3}\right)$ plot versus time.

effectively decreases linearly with time, thus validating the assumption of drying by diffusion. Moreover, from the slope, we determine an average experimental value of $j_{0}=(2.22 \pm 0.1) \times$ $10^{-10} \mathrm{~m}^{2} \mathrm{~s}$. This quantitative result is in agreement with numerical values from the literature ${ }^{12}$ (Table 1 ).

Eqn (3) can also be quantitatively compared to our experiments. In Fig. 8, we plot $\tau$ as a function of $V_{\mathrm{i}} R_{\mathrm{i}} / S_{\mathrm{i}}$. We deduce from the slope $3 /\left(2 j_{0}\right)$ that $j_{0}=(2.17 \pm 0.2) \times 10^{-10} \mathrm{~m}^{2} \mathrm{~s}^{-1}$, which is in good agreement with the value $2.22 \times 10^{-10} \mathrm{~m}^{2} \mathrm{~s}^{-1}$ deduced from the mass experiment and literature ${ }^{12}$ (Table 1).

We now propose to quantify the drying of liquid marble using a similar framework. The shape of a coated drop does not remain a spherical cap during drying as the surface buckles due the irreversible adsorption of the hydrophobic particles. ${ }^{7,17,25}$ However, assuming that: (i) the surface area of the drop remains constant after buckling $\left(S=S_{\mathrm{i}}\right.$ ); (ii) a radial local diffusion flux sets by the initial shape of the $\operatorname{drop} j_{0} / R_{\mathrm{i}}$, yields for the total evaporative flux: $\frac{\mathrm{d} V}{\mathrm{~d} t}=-\frac{j_{0}}{R_{\mathrm{i}}} S_{\mathrm{i}}$.

This gives after integration:

$$
V(t)=V_{\mathrm{i}}-\frac{j_{0}}{R_{\mathrm{i}}} S_{\mathrm{i}} t
$$

and

$$
\tau=\frac{1}{j_{0}} \frac{V_{\mathrm{i}} R_{\mathrm{i}}}{S_{\mathrm{i}}}
$$

Eqn (5) is similar to eqn (3), except for the numerical prefactor due to the assumption of conservation of the area of the interface after buckling.

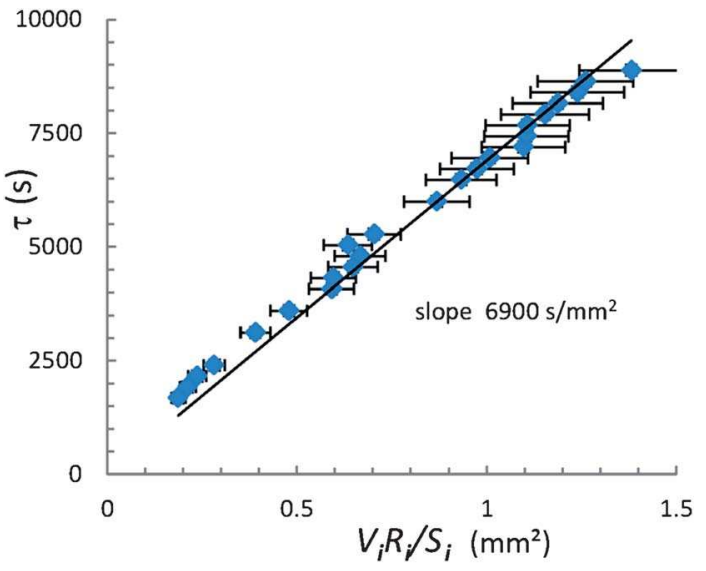

Fig. 8 Time of drying versus the ratio of volume time radius of curvature over surface area of the drop.

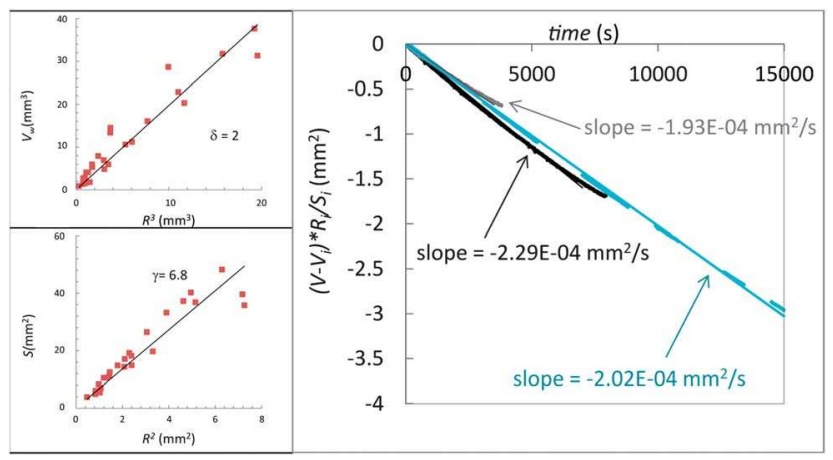

Fig. 9 Left: geometry of the coated droplet: initial volume (top) and initial surface area (bottom) as a function of initial radius of curvature; right: temporal evolution of the normalized volume $\left(V-V_{\mathrm{i}}\right) R_{\mathrm{i}} / S_{\mathrm{i}}$ for three coated drops of different initial volumes $\left(V_{\mathrm{i}}=7 \mathrm{~mm}^{3}, 27 \mathrm{~mm}^{3}\right.$ and $\left.67 \mathrm{~mm}^{3}\right)$.

As previously done for bare water drops with eqn (2) and (3), eqn (4) and (5) can be quantitatively compared to mass measurements of liquid marbles during drying and compared to the drying time measured by visualization.

Eqn (4) predicts that the volume of a coated liquid marble decreases linearly with time as previously shown in Fig. 5A. Moreover, we determine the initial water volume and the initial radius of curvature just before the buckling of the liquid marble surface (i.e. when the drop is still a spherical cap) (Fig. 7 left). Assuming $V_{\mathrm{i}}=\delta R_{\mathrm{i}}{ }^{3}$ and $S_{\mathrm{i}}=\gamma R_{\mathrm{i}}{ }^{2}$, we determine the geometrical constants $\delta$ and $\gamma$ (Fig. 9 left). These values are different from the previous geometrical constants $(\alpha$ and $\beta$ ) because coated drops and bare drops exhibit different contact angles. Another

Table 1 Measured values of the local evaporative flux $j_{0}$ from the literature and for the present data measured on bare and coated drops

\begin{tabular}{llllll}
\hline & Bare drops & & & & Coated drops \\
\cline { 2 - 3 } & Literature & Mass measurements & Visualization & & Mass measurements \\
\hline$j_{0}\left(10^{-10} \mathrm{~m}^{2} \mathrm{~s}^{-1}\right)$ & 2.5 & $2.2 \pm 0.1$ & $2.2 \pm 0.2$ & $2.1 \pm 0.2$
\end{tabular}




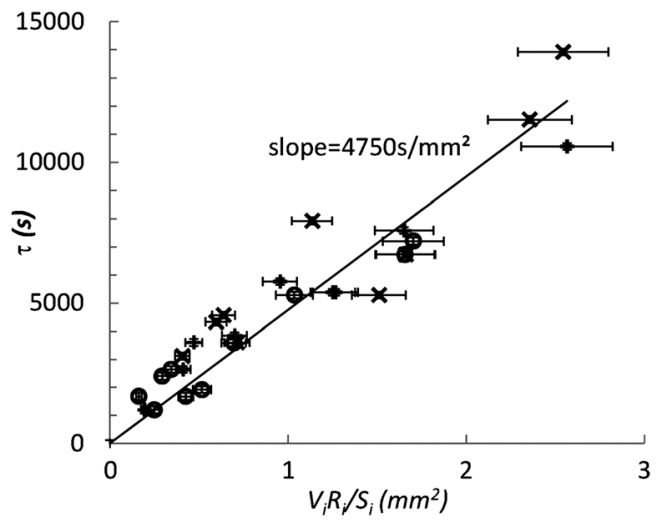

Fig. 10 Time of drying measured by the visualization of coated drops versus the ratio of volume time radius of curvature over the surface area of the drop. Different symbols correspond to different particles size: $40 \mu \mathrm{m}(*), 140 \mu \mathrm{m}(\times)$ and $500 \mu \mathrm{m}(+)$

source of deviation might be the excluded volume of the particles. We now test eqn (4) by plotting $\left(V-V_{\mathrm{i}}\right) R_{\mathrm{i}} / S_{\mathrm{i}}$ versus time (Fig. 9 right), we observe a relatively good collapse of the data for three liquid marbles of different initial volumes $\left(V_{\mathrm{i}}=7 \mathrm{~mm}^{3}\right.$, $27 \mathrm{~mm}^{3}$ and $67 \mathrm{~mm}^{3}$ ). From the slope we determine an average experimental value of $j_{0}=2.1 \pm 0.2 \times 10^{-10} \mathrm{~m}^{2} \mathrm{~s}^{-1}$.

Finally, we plot $\tau$ as a function of $V_{\mathrm{i}} R_{\mathrm{i}} / S_{\mathrm{i}}$ for three particle diameters in Fig. 10. Here also, we observe a relatively good collapse of the data as $\tau$ increases linearly with $V_{\mathrm{i}} R_{\mathrm{i}} / S_{\mathrm{i}}$. From the slope of Fig. 10 and eqn (5), we determine that $j_{0}=2.36 \pm 0.2 \times$ $10^{-10} \mathrm{~m}^{2} \mathrm{~s}^{-1}$. This value is in good agreement with the literature as for the one deduced from mass measurement of liquid marbles ( $c f$. Table 1$)$.

To summarize, drying of bare droplets or liquid marbles was determined considering mass measurement or the drying time yield $j_{0} \sim 2.1 \times 10^{-10} \mathrm{~m}^{2} \mathrm{~s}^{-1}$ as can be seen in Table 1 . This value, which is in good agreement with literature data, ${ }^{12}$ is independent of the coating of the drop by particles with a diameter around $100 \mu \mathrm{m}$. Thus it confirms recent results from the literature $\mathrm{e}^{13,14}$ and those obtained in the first part.

\section{Discussion}

Present results show that the droplets coated with a monolayer of hydrophobic particles dry faster than bare drops. Quantitative analyses explain this "speed up" by assuming that: (i) the monolayer does not modify the average diffusion flux over the interface; (ii) the area of exchange remains constant over drying due to incompressibility of the granular shell (constant surface area), in contrast to the decay of the area over time for the bare drop.

The first assumption is experimentally confirmed and is in agreement with recent experimental, computational and analytical work on evaporative fluxes from porous surfaces or through perforated masks of various geometry. ${ }^{13,14}$ The rates of water loss do not depend on the surface exposed to air but rather on the opening sizes and relative spacing of the pores. In particular, distributed small openings in the mask result in higher water loss rates than from equivalent large single openings. This variety of drying regimes can be explained by considering the competition between the local and average evaporation flux. At the local scale, evaporation is not uniform and depends on the local geometry around the interface. For example, when a mask with holes is positioned near or on a solid surface, the local evaporative flux is enhanced under the holes. These variations between free and hindered evaporation give rise to temperature and surface tension gradients across the film surface that induces a recirculating flow in the film. This effect, which may result in a local modulation of the thickness of the liquid, is used in evaporative lithography techniques..$^{26,27}$ However, at the global scale, the kinetics of drying may not be limited by the local evaporative flux but rather by the global geometry of the experiment. In such cases, the local inhomogeneities of vapour concentration are quickly equilibrated at a local scale, and thus do not influence the drying kinetics ( $c f$. Fig. 11). For the geometry of the monolayer of spheres, the distance between holes tends to zero because the spheres are tangent at one point (cf. Fig. 11). For this particular case, Shaehraeeni et al. predicted no modification of the evaporation rate due to the monolayer whatever the pore size. ${ }^{14}$ Thus, the drying rate measured at the global scale of the experiment does not depend on the local geometry of the interface.

The second assumption, concerning invariance of the surface area during drying, is expected because hydrophobic solid particles are irreversibly absorbed to the interface and are incompressible.

Our simple analysis predicts that the drying time of a bare drop is $50 \%$ larger than the drying time of a liquid marbles which is quite in agreement with experimental observations. This conclusion agrees with previous experimental data on drops coated with micrometric particles ${ }^{1,2}$ but not with others. ${ }^{3,4}$ Yet, the latter work reports on aggregation of particles. Thus,
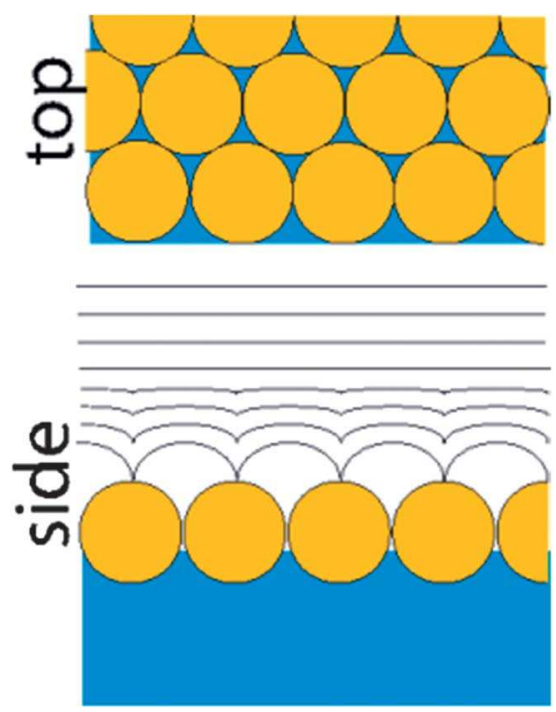

Fig. 11 Sketch of top and side views of a flat air-water interface coated with one monolayer of spherical particles. Black lines are illustration of the vapour isodensity above the interface inspired from ref. 14. 
particles at the surface of the drop may have arranged into several layers. Consequently, the presence of "thick" porous media around the interface may be responsible for the slowdown of the drying of such "multilayer" coated drops. Our results on drying through multilayers predict that the drying rate decays with the ratio $H / \Delta$ where $H$ is the height of the porous media and $\Delta$ the typical length scale of the interface ( $c f$. eqn (1)). Thus slowdown of drying is expected for a thick coating and/or small drops. Yet, when the contact diameter is of the order of $1 \mathrm{~mm}$ and the height of the porous media is $50 \mu \mathrm{m}$, we expect $A_{\mathrm{wg}} / A_{\mathrm{w}} \sim 0.7$ ( $c f$. Fig. 4), in agreement with ref. 4 , where at intermediate humidity a reduction of drying of $30-40 \%$ for drops of 4-5 $\mathrm{mm}^{3}$ coated with multilayers of hydrophobic powder, whose thickness can vary between 20 and $100 \mu \mathrm{m}$ was observed. For a large droplet and thin coating we assume that the incompressibility of the surface is dominant and speeds up the drying whereas for a small droplet and thick coating, the porous media has a dominant effect and thus slows down the drying.

It is noteworthy that the drying rate is independent of the particle sizes in the present case where the porous media is made of hydrophobic beads with sizes ranging from hundreds of micron to tens of nanometer. We assume that hydrophobic silica nanoparticles (R709 - Degussa) join into micrometric aggregates, thus, the pore size is always larger than the mean free path of vapor molecules which is of the order of $70 \mathrm{~nm}$. This result is in contrast to other systems made of hydrophilic silica nanoparticles (Ludox AS-40) and initially saturated with water, where slowdown of evaporation is associated with significant reduction of the diffusion coefficient in the Knudsen limit, as reported by Dufresnes et al. ${ }^{28}$ Moreover as particles are hydrophobic, vapor condensation is not expected at their surface whatever their size. Thus, in our experiment, the porous medium remains unchanged in regard to the vapor transfers in contrast to drying of saturated porous media. ${ }^{23}$ In addition, the thickness of the porous media does not evolve in time in opposition to the drying of drops made of colloidal suspensions or polymer solutions where the porous media is built at the periphery of the drop during drying. ${ }^{29}$ The present study on drying for water coated with hydrophobic particles is thus a very simple case that would help to describe more complex systems like the one cited above or others such as spray drying or foams Indeed, foams are subject to aging via diffusion of air through interfaces between bubbles. In the case of particle stabilized foams, ${ }^{30-32}$ the interface are covered of particles like in the present study.

\section{Conclusion}

The simple analysis presented in this work based on an average drying rate instead of a local drying rate gives good understanding of the process. Liquid marbles coated with one monolayer of hydrophobic micronic spherical particles dry faster than pure water droplet with a bare interface. This can be explained considering two effects: (i) water loss rate through a layer of dense micronic particles is almost the same as the one from bare interface. There is no reduction of the drying rate due to the particle coverage; (ii) yet, the solid particles lead to incompressibility of the interface whose area remains constant over the drying process. Thus, liquid marbles covered with one monolayer of particles, which exhibit more interface than liquid droplets, dry faster.

Nevertheless, liquid marbles covered with multilayer of particles are susceptible to dry slower than bare liquid droplet depending on the thickness of the coating compared to the size of the droplet. For example, the drying rate is halved for a coating thickness of one tenth of the drop size.

However, this analysis does not take into account the subtle mechanics of the interface under compression, which may exhibit fractures or invaginations. Thus, to describe quantitatively experiments with shells made of multilayers of particles, the modeling should take into account the porosity of shells as its fracture or the influence of local curvature of the surface after buckling on the drying rate. Indeed, we expect faster drying on a summit and lower drying in a valley due to the vapor saturation process in the valley. However such a modeling is far beyond the scope of this work.

\section{Acknowledgements}

We acknowledge Philippe Coussot for the fruitful discussion and thank him for introducing us to references on drying through porous media and partially covered surfaces. We also acknowledge Ludovic Pauchard for valuable discussions on drying phenomena in general and for pertinent comments on reading of this paper. Finally, F.R. is grateful to Narayanan Menon for outstanding discussions on granular rafts which, from one to another, has initiated this work. We thank Sylvie Cohen-Addad for giving us a sample of hydrophobic silica nanoparticles. We thank Olivier Pitois and Yacine Khidas for stimulating discussions and colleagues from Navier Laboratory for sharing experimental material in a convivial atmosphere.

\section{References}

1 C. Aberle, et al., Liquid marbles as thermally robust droplets: coating assisted Leidenfrost-like effect, Soft Matter, 2011, 7, 11314.

2 P. S. Bhosale, M. V. Panchagnula and H. A. Stretz, Mechanically robust nanoparticle stabilized transparent liquid marbles, Appl. Phys. Lett., 2008, 93, 034109.

3 M. Dandan and H. Y. Erbil, Evaporation rate of graphite liquid marbles: comparison with water droplets, Langmuir, 2009, 25, 8362-8367.

4 A. Tosun and H. Y. Erbil, Evaporation of PFTE liquid marbles, Appl. Surf. Sci., 2009, 256, 1278-1283.

5 P. Aussillous and D. Quéré, Liquid marbles, Nature, 2001, 411, 924.

6 P. Aussillous and D. Quéré, Properties of liquid marbles, Proc. R. Soc. London, Ser. A, 2006, 462, 973.

7 G. McHale and M. I. Newton, Liquid marbles: principles and applications, Soft Matter, 2011, 7, 5473-5481.

8 Y. Zhao, et al., Magnetic liquid marbles, their manipulation and application in optical probing, Microfluid. Nanofluid., 2012, 555-564. 
$9 \mathrm{H}$. Hu and R. G. Larson, Evaporation of a sessile droplet on a substrate, J. Phys. Chem. B, 2002, 106, 1334.

10 B. Sobac and D. Brutin, Thermal effect of the substrate on water droplet evaporation, Phys. Rev. E: Stat., Nonlinear, Soft Matter Phys., 2012, 86, 021602.

$11 \mathrm{H}$. Gelderblom, et al., How water droplets evaporate on a superhydrophobic substrate, Phys. Rev. E: Stat., Nonlinear, Soft Matter Phys., 2011, 83, 026306.

12 A. M. Cazabat and G. Guena, Evaporation of macroscopic sessile droplets, Soft Matter, 2010, 6, 2691-2612.

13 S. Assouline, K. Narkis and D. Or, Drying from partially covered water surfaces, Water Resour. Res., 2010, 46, W10539.

14 E. Shaehraeeni, P. Lehmann and D. Or, Coupling of evaporative fluxes from drying porous surfaces with air boundary layer: characteristics of drying from discrete pores, Water Resour. Res., 2012, 48, W09525.

15 S. S. Datta, H. Cheung Shum and D. A. Weitz, Controlled buckling and crumpling of nanoparticle-coated droplet, Langmuir, 2010, 26, 18612-18616.

$16 \mathrm{H}$. $\mathrm{Xu}$, et al., Shape and buckling transition in solidstabilized drops, Langmuir, 2005, 21, 10016-10020.

17 D. Vella, P. Aussillous and L. Mahadevan, Elasticity of an interfacial particle raft, Europhys. Lett., 2004, 682, 212-218.

18 R. Aveyard, et al., Structure and collapse of particle monolayers under lateral pressure at the octane/aqueous surfactant solution interface, Langmuir, 2000, 16(23), 8820-8828.

19 L. Pauchard and Y. Couder, Invagination during the collapse of an inhomogeneous spheroidal shell, Europhys. Lett., 2004, 66, 667-673.
20 N. Tsapis, et al., Onset of buckling in drying droplets of colloidal suspensions, Phys. Rev. Lett., 2005, 94, 018302.

21 L. E. Silbert, Jamming of frictional spheres and random loose packing, Soft Matter, 2010, 6, 2918-2924.

22 G. R. Farrell, K. M. Martini and N. Menon, Loose packings of frictional spheres, Soft Matter, 2010, 6, 2925.

23 P. Coussot, Scaling approach of the convective drying of a porous media, Eur. Phys. J. B, 2000, 557-566.

24 C. K. Ho and S. W. Webb, Gas transport in porous media, s.l: Springer, 2006.

25 P. Cicuta and D. Vella, Granular character of particle rafts, Phys. Rev. Lett., 2009, 102, 138302.

26 C. Parneix, et al., Dips and rims in dried colloidal films, Phys. Rev. Lett., 2010, 105, 266103.

27 D. J. Harris and J. A. Lewis, Marangoni effects on evaporative lithographic patterning of colloidal films, Langmuir, 2008, 24(8), 3681-3685.

28 E. R. Dufresnes, et al., Dynamics of fracture in drying suspensions, Langmuir, 2006, 22, 7144.

29 T. Okuzono, K. Ozawa and M. Doi, Simple model of skin formation caused by solvent evaporation in polymer solutions, Phys. Rev. Lett., 2006, 136103.

30 A. Stocco, et al., Particle-stabilised foams: structure and aging, Soft Matter, 2011, 7, 631-637.

31 A. Stocco, et al., Aqueous foams stabilized solely by particles, Soft Matter, 2011, 7, 1260.

32 D. Weaire and M. Vignes-Adler, New foams: fresh challenges and new opportunities, Curr. Opin. Colloid Interface Sci., 2008, 13(3), 141-149. 